\title{
MEASUREMENT OF THE ELECTRON LOSS CROSS SECTIONS FOR NEGATIVE HYDROGEN IONS ON CARBON AT 200 MEV
} Robert C. Webber and Carlos Hojvat*t

\section{SUMMARY}

The beam diagnostic equipment in the injection area of the Fermilab Booster accelerator has been used to measure the cross sections of electron loss for $\mathrm{H}^{-}$ ions on carbon. The details of the method are described. Preliminary results and comparison to theoretical calculations are presented.

\section{INTRODUCTION}

Capture or loss of electrons, the processes of charge exchange, take place when fast moving ions traverse a material. The study of the cross sections governing these processes is of both theoretical and practical interest. These phenomena can be utilized to obtain a desired admixture of charge states of a given atom.

In the field of accelerator physics, charge exchange processes are used in tandem accelerators, neutra? beams for heating of thermonuclear plasmas and for the method of charge exchange injection into cyclic accelerators.

$\mathrm{H}^{-}$charge exchange injection has recently become operational in the Fermilab Booster. 1 An integral part of the method is a stripping medium required to convert the injected $\mathrm{H}^{-}$ions into protons for capturing in the accelerator's orbit for acceleration.

The design of the stripping foils required know?edge of the electron loss cross sections at $200 \mathrm{MeV}$ to calculate the stripping efficiency. Although a large rumber of measurements for charge exchange cross sections do exist, ${ }^{2}$ the highest energy for which published results are available is $45 \mathrm{MeV} .{ }^{3}$ With the Fermilab Booster $\mathrm{H}^{-}$charge exchange injection system now operationa 1, the electron loss cross sections for $200 \mathrm{MeV}$ $\mathrm{H}^{-}$ions on carbon can be experimentally measured.

\section{METHOD}

When $\mathrm{H}^{-}$ions traverse a material, six charge exchange processes can occur. Three are electron loss reactions $(-1,0),(0,1),(-1,1)$ and three are electron pickup reactions $(1,0),(0,-1),(1,-1)$. For energies above $100 \mathrm{keV}$ the cross sections for electron pickup are very small and can be neglected. 2

Three differential equations describe the variation of the charge fractions with the foil thickness:

$$
\begin{aligned}
& \frac{d N^{-}}{d x}=-\left(\sigma_{-10}+\sigma_{-11}\right) N^{-} \\
& \frac{d N^{O}}{d x}=\sigma_{-10^{N}}-\sigma_{01} N^{O} \\
& \frac{d N^{+}}{d x}=\sigma_{-11} N^{-}+\sigma_{01} N^{O}
\end{aligned}
$$

where $x$ is the number of target atoms per $\mathrm{cm}^{2}, \sigma_{-1,0}$, $\sigma_{0,1}, \sigma_{-1,1}$ are the electron loss cross sections, and $\mathrm{N}^{+}, \mathrm{N}^{\circ}$ and $\mathrm{N}^{-}$the three charge fractions in the beam.

\footnotetext{
*Fermi NationaT Accelerator Laboratory, P. O. Box 500, Batavia, Illinois 60510 .
}

toperated by Universities Research Association, Inc., under contract with the U. S. Department of Energy.
Integrating (1):

$$
\begin{aligned}
& N^{-}=e^{-\left(\sigma_{-10}+\sigma_{-11}\right) x} \\
& N^{0}=\frac{\sigma_{-10}}{\sigma_{-10}+\sigma-11-\sigma_{01}}\left[e^{-\sigma_{01} x}-e^{-\left(\sigma_{-10}+\sigma_{-11}\right) x}\right] \\
& N^{+}=1-N^{-}-N^{O}
\end{aligned}
$$

The values of the three charge fractions can be measured experimentally as a function of the foil thickness.

A diagram of the Fermilab Booster $\mathrm{H}^{-}$injection system is shown in Figure 1. $200 \mathrm{MeV} \mathrm{H}^{-}$ions from a Linear accelerator pass through a carbon stripping foil at the injection point in the Booster. The electrons are stripped off the $\mathrm{H}^{-}$ions by the foil and the resulting beam of protons is set in the accelerator orbit by the second double magnet ORBMPII. Carbon was selected as the stripoing material because of its commercial availability, expected life time, low multiple scattering and relatively low cost."

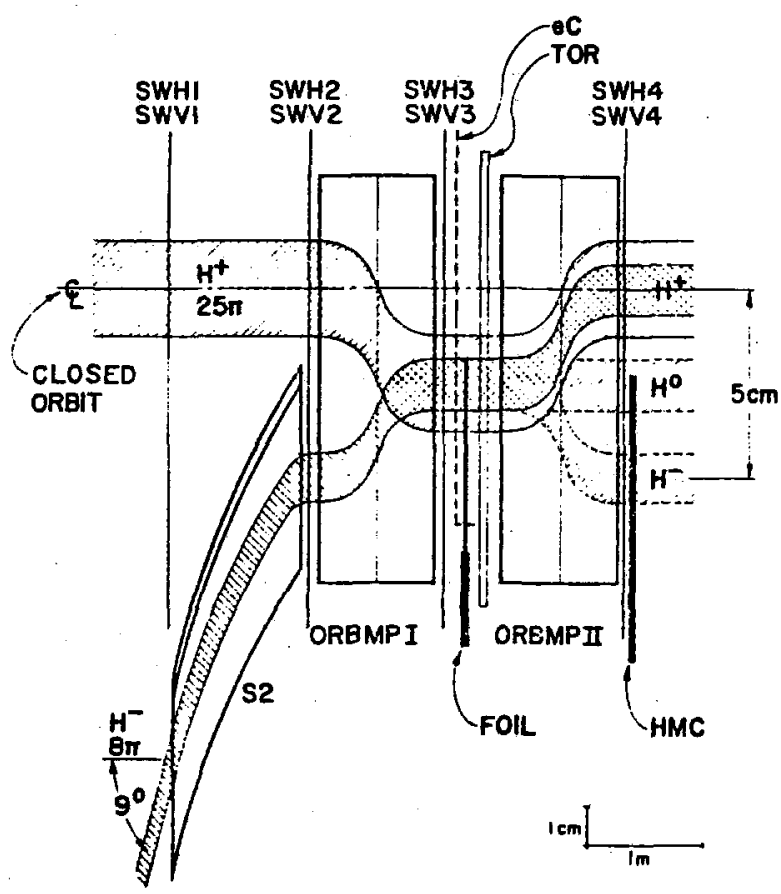

Fig. 1. Fermilab Booster injection area for Hoperation.

The method to determine the electron loss cross sections is based on utilizing the double magnet ORBMPII as an analyzer of the charge composition of the injected beam of $\mathrm{H}^{-}$ions after traversing the carbon foil.

The single wire scanners SWH4 and SWV4 are used to measure the charge fractions. A wire is moved across the aperture of the machine and a measurement of the beam profile is obtained. Downstream of ORBMPII the three charge fractions are separated horizontally but they overiap vertically. The horizontal scanner SliH4 determines the three fractions and the vertical scanner SWV 4 is used for monitoring as it measures the sum of 
the three charge states.

Beam current toroids in the $200 \mathrm{MeV}$ transport line provide normalization for fluctuations in the $\mathrm{H}^{-}$injected beam current.

\section{MEASUREMENTS}

$\mathrm{H}^{-}$beam currents between $14.5 \mathrm{~mA}$ and $31.8 \mathrm{~mA}$ for a duration of 2.8 us were injected into the Fermilab Booster in the normal manner. To prevent circulating beam from interfering with the measurements, the Booster guide field was run at a lower value so that stripped $\mathrm{H}^{-}$captured in the machine were lost before they could travel around the accelerator back to the injection area.

Foils of surface densities between $10 \mu \mathrm{gcm}^{-2}$ and $300 \mu \mathrm{gcm}^{-2}$ were utilized. The results of the horizontal scans with wire SWH4 are shown in Figure 2 for all the foils plus a scan with an empty frame for normalization. Separation between the different peaks is of the order of $2.0 \mathrm{~cm}$, comparable to the full width of the beams. The amount of a given charge fraction is assumed to be proportional to the peak voltage for that charge distribution. The error due to the overlap of neighboring distributions is smaller than other errors and has been neglected.

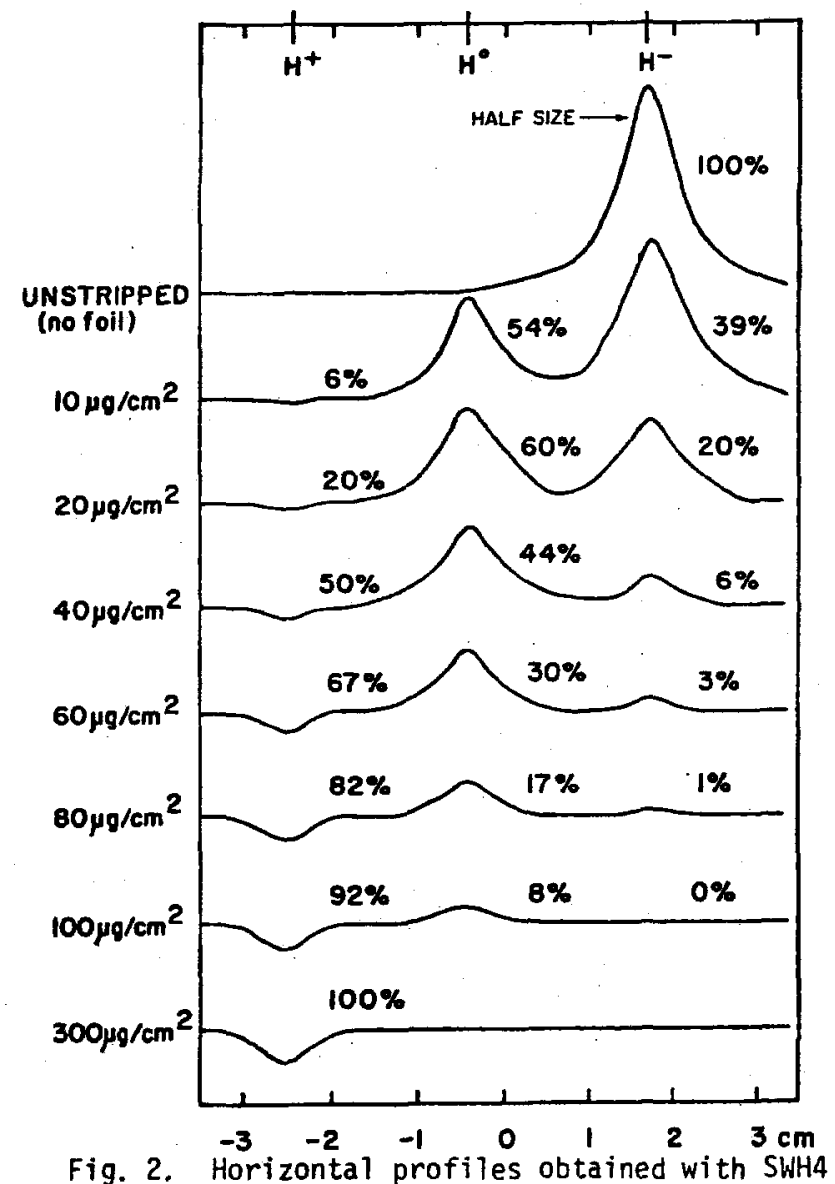

wire scanner from different foil thicknesses.

Different ions give rise to signals of different amplitudes as detected by the wire scanners. The $\mathrm{H}^{+}$ signal is due solely to secondary emission from the wire, the $H^{O}$ signal to capture of one electron plus secondary emission, and the $\mathrm{H}^{-}$signal to the capture of two electrons plus secondary emission. The wire scanners' sensitivity relative to the $\mathrm{H}^{-}$response was determined experimentally.
The sums of the three charge fractions should be equal to 1 for all foils. The relative sensitivity of the wire scanners to different ions is then obtained by minimizing the function:

$$
f=\Sigma_{i}\left|\frac{1}{v_{0}^{-}}\left(v_{i}^{-}+\alpha v_{i}^{+}+\beta v_{i}^{0}\right)-1\right|^{2}
$$

using $\alpha$ and $\beta$ as free parameters. $\alpha$ and $\beta$ are the inverse of the sensitivities to $\mathrm{H}^{+}$and $\mathrm{H}^{\circ}$ relative to $\mathrm{H}^{-}$ ions. $V_{i}^{-}, v_{i}^{+}$and $v_{i}^{O}$ are the voltages at the peak of the charge distributions for foil " $i$ ", and $V^{-}$is the peak voltage for the $\mathrm{H}^{-}$distribution with no foil (empty frame). From the minimization with the computer program MINUIT ${ }^{5}$ the values obtained are:

$$
\alpha=-13.5 \quad \beta=2.2
$$

The value of $\alpha$ is in good agreement with the measurements for the thicker foils which convert all H into $\mathrm{H}^{+}$ions. With the values above, the measured fractions of the beam for all the foils were calculated and included in Figure 2.

The sum of the three charge fractions for each foil is distributed as $1.00 \pm 0.035$ giving an idea of the errors involved.

The measured fractions $M^{-}$, $M^{\circ}$ and $M^{+}$and the theoretical distributions (2) are used to construct a $x^{2}$ function:

$$
F=\Sigma_{i}\left(\frac{M_{i}^{-}-N_{i}^{-}}{\sigma_{i}^{-}}\right)^{2}+\left(\frac{M_{i}^{o}-N_{i}^{0}}{\sigma_{i}^{o}}\right)^{2}+\left(\frac{M_{i}^{+}-N_{i}^{+}}{\sigma_{i}^{+}}\right)^{2}
$$

summed over all the foils " $i$ ". The errors $\sigma_{i}$ are determined for each measured charge fraction from the two following contributions. The foil thickness as measured by the supplier has an error of $\pm 10 \%$. The differential equations (1) are used to propagate the thickness error to an error in the fractions. Noise and non-linearities in wire signal amplifier electronics give an estimated error in the measured voltages of:

$$
(0.01)^{2}+\left(0.035 * V_{i}\right)^{2}
$$

The $x 2$ function was minimized with the computer program MINUIT using as parameters the three electron loss cross sections, resulting in the following values:

$$
\begin{aligned}
& \sigma_{-10}=(1.56 \pm 0.14) \times 10^{-18} \mathrm{~cm}^{2} \\
& \sigma_{01}=(0.60 \pm 0.10) \times 10^{-18} \mathrm{~cm}^{2} \\
& \sigma_{-11}=(-0.08 \pm 0.13) \times 10^{-18} \mathrm{~cm}^{2}
\end{aligned}
$$

for a final value $F=18.6$ for 9 degrees of freedom. The theoretical distributions calculated with these values, setting $\sigma_{-11}=0$ are plotted in Figure 3 together with the experimental results. 


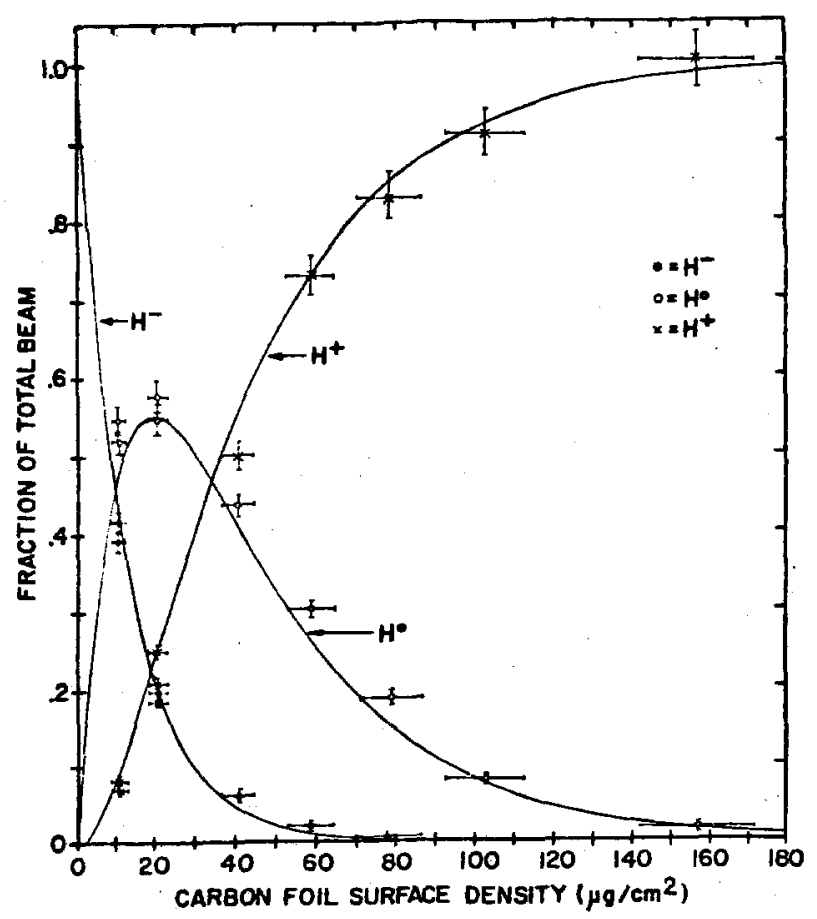

Fig. 3. Comparison of measured charged fractions to the theoretical curves.

\section{DISCUSSION}

The value for $\sigma_{-11}$ is consistent with zero. The method, with the errors as presently understood, is not sensitive enough to determine the value of this cross section.

Figure 4 summarizes the presently available data and recent theoretical calculations for comparison with our resuits.

The $\sigma_{01}$ results are those of Acerbi et al. between 22 and $45 \mathrm{MeV} .{ }^{3}$ The plotted values for carbon were obtained from their measurements for $\mathrm{CH}_{4}$ and $\mathrm{H}_{2}$ by $\sigma_{01}(\mathrm{C})=\sigma_{01}\left(\mathrm{CH}_{4}\right)-2 \sigma_{01}\left(\mathrm{H}_{2}\right)$. The rest of the experimental data are for oxygen, nitrogen and air. $5,7,3,9,10$ Results of recent theoretical calculations by Gillespie for carbon are included. 11

Very good agreement is observed between our results for $\sigma_{0}$ and the theoretical calculation. For $\left(\sigma_{-1} 0^{+\sigma_{-}} 11\right)$ the agreement is within two standard deviations. The theoretical curves seem to follow the cross section's dependence with energy very closely, for this energy region.

\section{ACKNOWLEDGMENTS}

The authors wish to acknowledge the support of the Fermilab Accelerator Division and their colleagues in the Booster Group for allowing the use of valuable accelerator study time for these measurements. Discussion of theoretical calculations and access to a summary of experimental data by G. H. Gillespie is gratefully acknowledged.

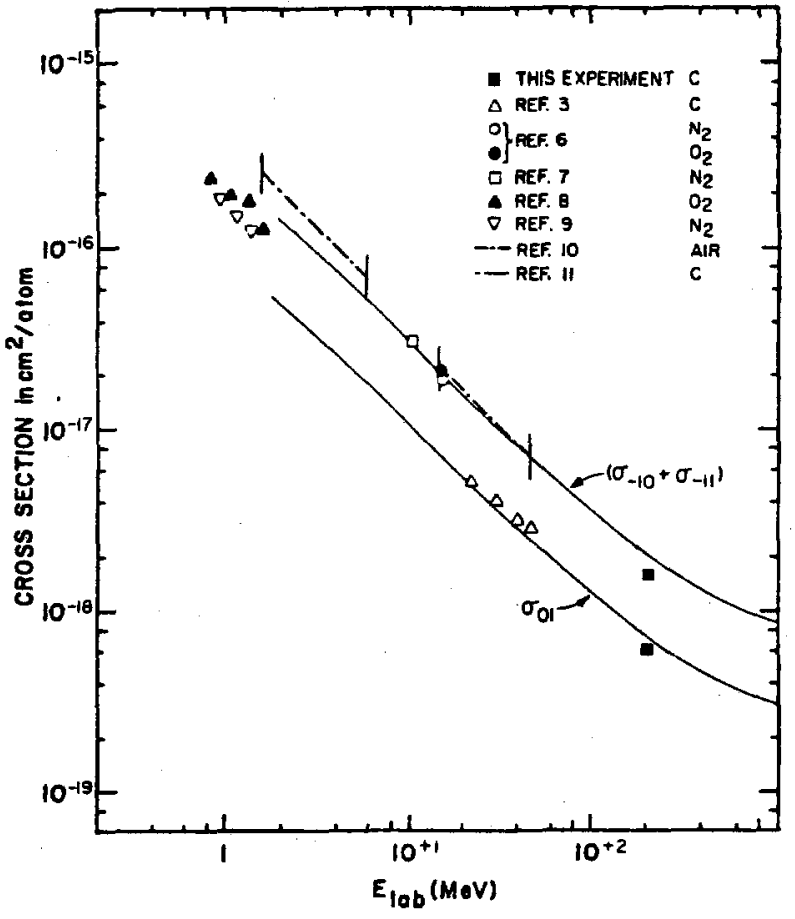

Fig. 4. Comparison of the results from this experiment with previous results and theoretical predictions.

\section{REFERENCES}

1. C. Hojvat et al., "The Multiturn Charge Exchange Injection System for the Fermilab Booster Accelerator", this conference.

2. H. Tawara and A. Russek, Rev. Mod. Phys. 45, 178, 1973.

3. E. Acerbi et al., Lett. Nuovo Cimento 10, 598, 1974.

4. C. Hojvat, M. Joy and R. C. Webber, "Stripping Foils for Multiturn Charge Exchange Injection into the Fermilab Booster", this conference.

5. F. James and M. Roos, "Minuit", CERN Computer Program Library, D506/D516.

6. R. Smythe and J. W. Toevs, Phys. Rev. 139, A15, 1965.

7. K. H. Berkner, S. N. Kaplan, and R. V. Pyle, Phys. Rev. 134, A1461, 1964.

8. P. H. Rose, R. J. Connor and R. P. Bastide, Bull. Am. Phys. Soc. 3, 40, 1958. Actual data are given in review articles by S. K. Allison, Rev. Mod. Phys. 30 , 1137, 1958; and by $H$. Tawara and $A$. Russek, op. cit.

9. G. I. Dimov and V. G. Dudnikov, Zh. Tekh, Fiz. 36, 1239, 1966. (Sov. Phys. - Tech. Phys. 11, 919, 1967.)

10. J. H. Fremlin and V. M. Spiers, Proc. Phys. Soc. Lond. A 68, 398, 1955.

11. G. H. Gillespie, Phys. Rev. A 16, 943, 1977; and private communication. 